\title{
Psychometric parameters of the Persian version of the Brief COPE among wives of patients under hemodialysis
}

\author{
Tahereh Ashktorab $^{1}$, Nayereh Baghcheghi ${ }^{1 *}$, Naimeh Seyedfatemi ${ }^{2}$, Ahmadreza Baghestani $^{3}$ \\ Received: 18 Oct 2016 \\ Published: 11 Mar 2017
}

\begin{abstract}
Background: The Brief COPE is widely validated and popularly used in assessing coping strategies in major life stressors on various populations. This study carried out to determine the validity and reliability of the Persian version of the Brief COPE among wives of patients under hemodialysis.

Methods: The Brief COPE was translated into Persian language according to the standard method and the psychometric properties of the instrument were assessed among 212 wives of patients under hemodialysis in Tehran, Iran, in 2016. Content validity was established, by 15 expert opinions. Face validity was determined by respondents and expert opinion. Construct validity was analyzed through confirmatory factor analysis. The Cronbach's alpha coefficient and intraclass correlation coefficient were used to determine the internal consistency and test-retest reliability, respectively.

Results: All 28 Items of Brief COPE scale had content validity index greater than 0.7 , suggesting a good validity value of the items in terms of relevancy (range $0.8-0.94$ ). The wording of some items was modified to make them more readily understood by the participants, although the core meaning of each item was kept intact and to observe Iranian cultural adaptation; two items were adjusted. Result of confirmatory factor support 14 factor structure of the scale has good fit. Cronbach's alpha coefficient was acceptable for the total scale $(\alpha=0.77)$ and for subscales (range $0.7-0.91)$. The intraclass correlation coefficient was acceptable for scale $(r=0.76, P$ val$\mathrm{ue}=0.001)$.

Conclusion: The Persian translation of the Brief COPE is a valid and reliable instrument to determine coping strategies in women living with the husband on hemodialysis.
\end{abstract}

Keywords: Cope, Hemodialysis, Validity, Reliability

Copyright $₫$ Iran University of Medical Sciences

Cite this article as: Ashktorab T, Baghcheghi N, Seyedfatemi N, Baghestani A. Psychometric parameters of the Persian version of the Brief COPE among wives of patients under hemodialysis. Med J Islam Repub Iran. 2017 (11 Mar);31:20. https://doi.org/10.18869/mjiri.31.20

\section{Introduction}

Chronic kidney disease (CKD) is a growing and major health problem in the world, which is featured with high rate of morbidity and mortality (1). In 2013, the prevalence of hemodialysis patients was reported in the world and in Iran 1,500,000 and 25,000, respectively (2). According to the global burden of disease report, end-stage renal disease was ranked $18^{\text {th }}$ in the list of causes of total number of deaths worldwide in 2010 , while was ranked 27th in 1990. This degree of the climb up within the list was second compared to HIV and AIDs (3). It is predicted that by 2020 , there will be 1200 cases of chronic kidney disease per million population (4). It has become increasingly apparent that hemodialysis not only affects the pa-

Corresponding author: Dr Nayereh Baghcheghi, baghcheghinayereh@gmail.com

1. School of Nursing \& Midwifery, Shahid Beheshti University of Medical Sciences, Tehran, Iran.

2. Nursing Care Research Center, Iran University of Medical Sciences, Tehran, Iran

3. School of Paramedical Sciences, Shahid Beheshti University of Medical sciences, Tehran, Iran. tient, but also disrupts the entire family unit, including lifestyle of the patients and those close to them such as the spouse. Hemodialysis patients and their families have little information to help them prepare for and contend with their long hemodialysis travel (5). Both patient and their family suffer the consequences of the disease; this means that the relatives and the family, as a social system, must be under focus by the researchers (6). A key factor in discontinuing dialysis is that the patient may develop the perception that they are a burden to their family members (7). Effects of the chronic burden experienced by family caregivers can result in physical and mental disturbances and also influence the quality of care directly provided to

$\uparrow$ What is "already known" in this topic:

The Brief COPE is widely used as a coping measure and to evaluate coping strategies in different illnesses. Validated versions of this questionnaire in a variety of languages are currently available.

$\rightarrow$ What this article adds:

The Persian translation of the Brief COPE is a valid and reliable instrument, therefore, Iranian researchers can use the Brief COPE as a valid and reliable tool to measure coping strategies in Iranian wives of patients under hemodialysis. 
patients (8). What is usually missed is providing support for the patients' families, which is required to prevent overburdening or mental issues. Investigations should focus on how chronic kidney disease and dialysis affects the family life and on how the family can be supported in coping with this situation and its consequences (6).

There are numerous studies on psychological aspects of chronic kidney disease hemodialysis patients, but only a few investigators have attempted to describe the process of coping in women living with the husband on hemodialysis. Men and women's role as caregivers and the differences between them have be studied by researchers. Normally, different patterns of supporting behaviors are demonstrated by men and women. These patterns fit a gender-based division of labor. For instance, women tend to handle most of day-to-day, personal, and hands-on care. This means, women usually face a greater negative repercussions of caregiving, which is known as burden or stress (9). With the rapidly growing number of elderly patients with end-stage renal disease (ESRD), burdens on their families to provide care and support have increased. Emotional dimensions of patients under hemodialysis and their caregivers (particularly female spouses) are important predictors of burden. Social support and psychological interventions should be taken into account to better caregiver life and patient outcomes (8).

Coping with a disease is rarely an individual work, so that the partner suffers the consequences of illness of his/her partner (10). According to Lazarus and Folkman, coping is "constantly changing cognitive and behavioral efforts to manage specific external and/or internal demands that are taxing or exceeding the resources of a person" (11). To put it another way, coping has been defined as an attempt to deal with demands that exceed the resources of the person and effective coping mechanisms may neutralize stressors, reducing their effect on disease progression (12). Coping with the stresses of chronic illness is crucial to determining changes in health-related quality of life(13). It also represents how well patients adjust to chronic diseases (14).

Several tools have been developed to measure coping, including Folkman and Lazarus' Ways of Coping Questionnaire with 66 statements (15) and Carver' COPE designed the 60-item scale (16) Endler and J Parker' Coping inventory for stressful situations, consisting of 48 items (17). One frequently used coping scale is the COPE by Carver (1989) at the University of Miami, which assesses 15 theoretically derived coping strategies using four item allocated to the subscales viz. positive reinterpretation and growth, Mental disengagement, Focus on and venting of emotions, Use of instrumental social support, Active coping, Denial, Religious coping, Humor, Behavioral disengagement, Restraint, Use of emotional social support, Substance use, Acceptance, Suppression of competing activities and Planning(16). A major difficulty of three mentioned questionnaires is the length and thus the potential for the subject burden. Carver (1997) introduced the Brief COPE, which is a short version of the COPE, to reduce subject burden and address other limitations of the COPE. This scale is comprised of 28 items that assess 14 coping strategies based on a four-point Likert scale ("I haven't been doing this at all", "I've been doing this a little bit", "I've been doing this a medium amount", and "I've been doing this a lot"), so that two items are allocated to each one of the 14 subscales; the subscales are active coping, planning, positive reframing, acceptance, humor, turning to religion, using emotional support, using instrumental support, self-distraction, denial, venting, substance use, behavioral disengagement, and self-blame.(3, 18). The Brief COPE is widely used as a coping measure and cited by more than 900 articles by August 2011(19). The Brief COPE is used to evaluate coping in different illnesses, such as perinatal depression(20), cystic fibrosis (21), and mental illness (22). Validated versions of the Brief COPE in Brazilian(23), Spanish (Spain) (24), Korean (South Korea) (25), French (France) (26), Portuguese (Portugal) (27), Greek (Greece) (28) and Tamil (India) (29) are currently available.

Many women are living with the husband on hemodialysis who have to cope with stressful issues in Iran. As the Brief COPE was not investigated in this context, and in view of the concerns mentioned above, the present study is an attempt to translate and adapt the original Brief COPE to Persian and evaluate the psychometric parameters of the translated version as well.

\section{Methods}

\section{Study Design and Participants}

The present study was a cross-sectional psychometric evaluation that was conducted on 212 wives of patients under hemodialysis, in Tehran, Iran, in 2016. The minimum sample size usually is considered to be 5 to 20 samples per each item for the studies of the construct validation of instruments (30). Two hundred and twelve participants were selected through nonprobability sampling among available individuals' method in hospitals of Shahid Beheshti University of Medical Sciences ((Ayatollah) Taleghani Educational Hospital, Shohadaye Tajrish Educational Hospital, Shahid Labbafi Nedjad Educational Hospital, Shahid Modarress Educational Hospital, Imam Hossein Educational Hospital).

Following approval by the university Research Ethics Board, the researcher met hemodialysis patients and their wives at hemodialysis ward in hospitals of Shahid Beheshti University of Medical Sciences to distribute consent forms and questionnaires.

Since some of the participants are not literate, for this group these items were rephrased into questions so that the questionnaire became interviewer based.

The inclusion criteria included: willingness to participate in the study, being at least 6 months under hemodialysis in husbands(31,32), no known case of psychiatric disorders in women and their husbands according to their self-report $(31,32)$, and Persian as their native language. If needed, spoken information about completing questionnaire was considered for low literacy levels participants.

Of the 212 recruited participants, the mean age was 54.2 \pm 13.7 years $($ Range $=31-65), 72 \%$ were housewives, and mean duration of disease was $5.33 \pm 4.3$ years. In terms of education level, 4.71 were illiterate, $58.8 \%$ had primary 
education, $15.09 \%$ had junior high school education, $16.98 \%$ had high school diploma, and $5.18 \%$ had a college degree.

\section{Data collection}

Following extensive consultation with experts and comprehensive review of the related literature, the original version of the questionnaire was selected to be used in the study. The lead author's consent to use the original scale was secured beforehand (33). Here, a forward-backward translation method was followed to develop the Persian version of the questionnaire on the basis of Wild et al. 's approach(33). Data provided by the corresponding author with the help of three other authors that one of the authors specializes in Biostatistics.

\section{Content validity}

Content validity of an assessment instrument is a subjective judgment by experts about the degree of the relevant and representative construct (34). The most widely reported method for content validity is the content validity index (35). We collected and analyzed qualitative and quantitative viewpoints of 15 expert faculty members to ensure that the instrument has content validity. Content validity Index was determined based on relevancy. The relevance criterion was assessed at four levels: 1 [not relevant], 2[somewhat relevant], 3[quite relevant], 4[highly relevant]). According to the expert opinion, items with more than 0.79 validity were considered as proper statements while items with a CVI of 0.7-0.79 were regarded as the items requiring modification (36).

\section{Face validity}

Face validity or appearance validity is not considered as validity as far as measurement principles are concerned. In fact, it does not consider what to assess, but it focuses on the appearance of the instrument (37). To conduct face validity researchers used respondents and experts' viewpoints(38). Difficulty, ambiguity, and misinterpretations of items, and/or incomprehensibility of the meaning of words are the issues discussed and modified.

\section{Construct validity}

In order to establish construct validity, 212 women living with the husband on hemodialysis were recruited from hemodialysis ward in Hospitals of Shahid Beheshti University of Medical Sciences through nonprobability sampling among available individuals' method. With regard to factorial structure of the translated version of the Brief COPE, Confirmatory factor analysis (CFA) was carried out in LISREL (version 8.8) (39). In general, CFA has a strong theoretical and empirical bases that makes the investigator able to determine a hypothesized factor structure beforehand and then test it. In CFA, the researcher determines a fix number of factors, if the factors are correlated or not, and how to measure the factors(40). Global fit was measured through measuring 1) the goodness of fit index (GFI), which uses a ratio of total squared discrepancies between the observed and population variance, 2) root mean square error of approximation (RMSEA), which measures the mean discrepancy between the observed covariances and those determined by the model based on degree of freedom, 3) comparative fit index (CFI), which determines improvement in fit of the hypothesized model in comparison with a fully independent model, and 4) the standardized root mean residual (SRMR), which is used to determine the degree to which a priori structure that regenerates the data is evaluated (41). Usually, relative $\chi 2$ fit index is set at 3:1 ratio, while some authors prefer a higher ratio of around 5:1(42). In this regard, RMSEA of $\leq 0.05$ is interpreted as a good fit; $>0.05$ to $\leq 0.08$ as a reasonable fit; $>0.08$ to $\leq 0.1$ as mediocre; and $>0.1$ as poor. Comparative Fit Index (CFI) and General Fit index (GFI) normed fit index (NFI), the non-normed fit index (NNFI) equal to or greater than 0.9 were taken as a good fit. Moreover, standardized root mean residual values (SRMR) less than or equal to 0.05 were taken as good fit (43).

\section{Reliability}

The reliability of the Persian Brief COPE was measured through obtaining its internal consistency and test-retest method. Cronbach's alpha was used to measure internal consistency and a reliability coefficient of 0.70 or higher was considered adequate reliability (44). Carver reported a level of 0.5 for acceptability (in two items subscales which have only 2 items per subscales) (18), although 0.7 to 0.8 has been recommended by others $(44,45)$ and is conventionally considered reliable for research purposes. intra-class correlation coefficient (ICC) was used to measure test-retest reliability or reproducibility of responses obtained at the two-time points, where 0.60 was interpreted as marginal, 0.70 as acceptable and $0.80<$ was considered as high correlation (46). In order to assess test-retest reliability, the Persian version of Brief COPE was administered in 10-14 days' intervals to a sample of 20 (47) wives of patients under hemodialysis.

\section{Statistical analysis}

Statistical analysis was done in SPSS version 18 considering a significance level of $\mathrm{p}<0.05$ and LISREL 8.80 . Normality assumption was tested using KolmogorovSmirnov test, and as a result, data were normally distributed. The analysis showed a normal distribution.

\section{Ethical consideration}

The ethical permission for this study was secured from the Ethics Committee of the Shahid Beheshti University of Medical Sciences (Code of ethics: phnm.1395-395); and permission for translation, modification, and use of the Brief COPE was obtained from Carver.

\section{Results}

Of the 235 questionnaires administered to the sample, 212 respondents completed and returned the questionnaires; thus, the response rate was $90 \%$.

All 28 Items had CVI greater than 0.70 suggesting a good validity value of the items in terms of relevancy (range 0.8 - 0.94); therefore, no item was eliminated from the questionnaire. Based on the qualitative viewpoints of experts, some recommendations were approved and to 
Table 1. Goodness of Fit indices of Persian version of the Brief COPE model

\begin{tabular}{llll}
\hline Fit Index type & Observed value & Acceptable value & Fit level \\
\hline Relative $\chi^{2}$ fit index & 1.06 & $<3$ & Good Fit \\
GFI & 0.93 & $\geq 0.9$ & Good Fit \\
IFI & 0.99 & $\geq 0.9$ & Good Fit \\
CFI & 0.97 & $\geq 0.9$ & Good Fit \\
NFI & 0.9 & $\geq 0.9$ & Good Fit \\
NNFI & 0.99 & $\geq 0.9$ & Good Fit \\
SRMR & 0.04 & $\leq .05$ & Good Fit \\
RMSEA & 0.02 & $\leq .05$ & Good Fit \\
\hline GFI: Goodness of Fit Index, IFI: Incremental Fit Index, CFI: Comparative Fit Index, NFI: Normed Fit Index, SRMR: standardized Root Mean Square Residual, RMSEA: Root Mean Square Error of Approximation
\end{tabular}

observe Iranian cultural adaptation, In the fourth and the eleventh items of the original scale ("I've been using alcohol or other drugs to make myself feel better" and "I've been using alcohol or other drugs to help me get through it") the terms "alcohol or other drugs" were changed into "drugs and other things". The wording of some items was modified to make them more readily understood by the participant, although the core meaning of each item was kept intact.

Model fit indices are illustrated in Table 1, and goodness of fit of the model is shown by confirmatory factor analysis.

Cronbach's alpha was acceptable both for the whole scale $(\alpha=0.77)$ and for subscales (range $0.7-0.91)$. These are presented in Table 2. The ICC for the 28 item Brief COPE scale was equal to $0.76(\mathrm{p}=0.001)$, indicating acceptable test-retest reliability.

\section{Discussion}

As far as the authors know, this study is the first to psychometrically validate the Brief COPE within a sample of women living with the husband on hemodialysis and the first to psychometrically validate the Persian translation of the Brief COPE regardless of the type of population in Iran. Previous research has suggested that measures of coping behaviors should be validated for particular patient groups before they can be confidently applied to those groups (48). The present study showed evidence for good psychometric parameters of the Persian translation of the Brief COPE, after a few changes in wording and format base on cultural adaptation.

Alcohol use strategy that is used in the fourth and eleventh items in an original scale is not compatible with Ira-

Table 2. Reliability coefficients of the total scale and every sub-scales using Cronbach's alpha formula

\begin{tabular}{ll}
\hline Sub-scales & $\begin{array}{l}\text { Internal consistency } \\
\text { (Cronbach's Alpha) }\end{array}$ \\
\hline Active coping & 0.7 \\
Planning & 0.80 \\
Positive reframing, & 0.81 \\
Acceptance & 0.76 \\
Humor & 0.91 \\
Turning to religion & 0.78 \\
Using emotional support & 0.85 \\
Using instrumental support & 0.87 \\
Self-distraction & 0.77 \\
Denial & 0.85 \\
Venting & 0.77 \\
Substance use & 0.9 \\
Behavioral disengagement & 0.91 \\
Self-blame & 0.78 \\
Total & 0.77 \\
\hline
\end{tabular}

nian culture; therefore, the two mentioned items, were modified. According to Islamic laws, using any intoxicants (specifically, alcoholic beverages) is generally forbidden and this code is repeatedly mentioned in the Holy Qur'an. Following this religious code, alcohol beverages are illegal in Iran

In our study, the reliability of the scale was satisfactory, and analysis suggested that the Persian translation of the Brief COPE shown satisfactory internal consistency; this reflects that the tool is consistent and reproducible in identifying coping strategies in women living with the husband on hemodialysis. Moreover, in terms of reliability, the tool is comparable with the original Brief COPE that is evaluated by the Carver(18) and the Malay Brief COPE among adolescents (49), and Brief COPE among medical student(50) and Monzani study (51). All of the subscales showed acceptable internal consistency; this also indicates reliability of the Persian Brief COPE. This is consistent with the Monzani study (51).

In our study, confirmatory factor analysis suggested goodness of fit of the 14-factor model with the data, which supports the theoretically based 14-factor structure introduced by Carver (18). Dias (2009) carried out a CFA to evaluate the psychometric parameters of the Portuguese translation of the Brief COPE. Findings supported good psychometric properties of the instrument (52). Monzani et al. (2015) employed confirmatory factor analysis and compared five models of the Brief COPE (Theoretically based 14-factor structure, 9-factor, 11-factor, 4 and 7factor structure the Brief COPE). The results indicated that the theoretically based 14-factor was the only model with good fit (51). Still, some studies have shown that several scales can be clustered together into broader factors. For instance, Kapsou et al. examined construct validation of the Brief-COPE with a Greek-speaking subjects and CFA confirmed a good fit for the eight-factor model (28). The Hagan et al. study (2016) demonstrated a satisfactory psychometric parameters of the Brief COPE in patients newly diagnosed with incurable cancer. This study largely supported a seven-factor solution matching the original subscales, with the exception of the behavioral disengagement subscale that had an extremely low internal consistency and a weak factor loading. This pattern may be related to patients' hesitancy to endorse items that suggest they are "giving up" trying to improve their lives with cancer (a term used within both behavioral disengagement items) when newly confronted with an incurable cancer diagnosis (53).

Measurement of coping can be affected by culture, language, and acculturative status(54). Given the lack of 
instruments in Persian, appropriate assessment of Persianlanguage people is difficult. The Brief COPE is a practical, simple measure that quickly assesses an individual's coping strategies. Such information may help clinicians assist patients to cope more adaptively by using effective strategies to enhance the quality of life and $\operatorname{mood}(53)$.

Present study confirms that the Brief COPE can measure 14 unique strategies including Self-distraction, Active coping, Denial, Substance use, Use of emotional support, Use of instrumental support, Behavioral disengagement, Venting, Positive reframing, Planning, Humor, Acceptance, Religion, and Self-blame. Given this, future researches and clinical psychologists can use these 14 distinct scores of coping responses. Generally, the findings indicate that the Persian translation of the Brief COPE is a valid and reliable instrument to determine coping strategies in women whose husband is on hemodialysis.

The limitation of this study is the nonprobability sampling among available individuals; this bias can affect the accuracy of the study. However, the present study gives useful information for the future works. Further similar studies to use Brief COPE through randomized sampling are important to determine its capability of measurement.

\section{Conclusion}

It is imperative to have a valid and reliable assessment of coping strategies in wives of patients under hemodialysis. The present study provides an empirically precise and theoretical supported structure model of Brief COPE for assessing coping strategies in Persian wives of patients under hemodialysis. Therefore, Iranian researchers and clinicians can use the Brief COPE as a valid and reliable tool to measure coping in Iranian wives of patients under hemodialysis.

\section{Acknowledgements}

This article is part of the $\mathrm{PhD}$ nursing thesis at Shahid Beheshti School of Nursing and Midwifery is in Tehran, in 2015. (Thesis serial number: 395). The authors would like to thank all of the women who took part in this study

Conflict of Interests: The authors declare that they have no competing interests.

\section{References}

1. Ye J-J, Zhou T-B, Zhang Y-F, Wang Q, Su Y-Y, Tang J-M, et al. Levels of vitamin D receptor and CYP24A1 in patients with endstage renal disease. Afr Health Sci. 2016;16(2):462-7.

2. Shahgholian N, Yousefi H. Supporting hemodialysis patients: A phenomenological study. Iran J Nurs Midwifery Res. 2015;20(5):626.

3. Lozano R, Naghavi M, Foreman K, Lim S, Shibuya K, Aboyans V, et al. Global and regional mortality from 235 causes of death for 20 age groups in 1990 and 2010: a systematic analysis for the Global Burden of Disease Study 2010. Lancet. 2013;380(9859):2095-128.

4. Zheng J, You L-M, Lou T-Q, Chen N-C, Lai D-Y, Liang Y-Y, et al. Development and psychometric evaluation of the Dialysis patientperceived Exercise Benefits and Barriers Scale. Int J Nurs Stud. 2010;47(2):166-80.

5. Lin C-C, Han C-Y, Pan I-J. A qualitative approach of psychosocial adaptation process in patients undergoing long-term hemodialysis.
Asian Nurs Res. 2015;9(1):35-41.

6. Schipper K, Abma TA. Coping, family and mastery: top priorities for social science research by patients with chronic kidney disease. Nephrol Dial Transplant. 2011;26(10):3189-95.

7. Ashby M, op't Hoog C, Kellehear A, Kerr PG, Brooks D, Nicholls K, et al. Renal dialysis abatement: lessons from a social study. Palliat Med. 2005;19(5):389-96.

8. Belasco AG, Sesso R. Burden and quality of life of caregivers for hemodialysis patients. Am J Kidney Dis. 2002;39(4):805-12.

9. Neal MB, Ingersoll-Dayton B, Starrels ME. Gender and relationship differences in caregiving patterns and consequences among employed caregivers. Gerontologist. 1997;37(6):804-16.

10. Kenny DA, Cook W. Partner effects in relationship research: Conceptual issues, analytic difficulties, and illustrations. Pers Relatsh. 1999;6(4):433-48.

11. Lazarus RS, Folkman S. Stress, appraisal, and coping: Springer publishing company; 1984.

12. Folkman S. Stress: Appraisal and coping. Encyclopedia of behavioral medicine: Springer; 2013. p. 1913-5.

13. Sprangers MA, Schwartz CE. Integrating response shift into healthrelated quality of life research: a theoretical model. Soc Sci Med. 1999;48(11):1507-15.

14. de Ridder D, Geenen R, Kuijer $R$, van Middendorp $H$. Psychological adjustment to chronic disease. Lancet. 2008;372(9634):246-55.

15. Folkman S, Lazarus RS. Manual for the ways of coping questionnaire: Consulting Psychologists Press; 1988.

16. Carver CS, Scheier MF, Weintraub JK. Assessing coping strategies: a theoretically based approach. J Pers Soc Psychol. 1989;56(2):267.

17. Endler NS, Parker J. Coping inventory for stressful situations: Multi-Health systems Incorporated; 1990.

18. Carver CS. You want to measure coping but your protocol'too long: Consider the brief cope. Int J Behav Med. 1997;4(1):92-100.

19. Kasi PM, Naqvi HA, Afghan AK, Khawar T, Khan FH, Khan UZ, et al. Coping styles in patients with anxiety and depression. ISRN psychiatry. 2012;2012.

20. de Tychey C, Spitz E, Briançon S, Lighezzolo J, Girvan F, Rosati A, et al. Pre-and postnatal depression and coping: a comparative approach. J Affect Disord. 2005;85(3):323-6.

21. Wong M, Heriot S. Parents of children with cystic fibrosis: how they hope, cope and despair. Child Care Health Dev. 2008;34(3):34454.

22. Meyer B. Coping with severe mental illness: Relations of the Brief COPE with symptoms, functioning, and well-being. J Psychopathol Behav Asses. 2001;23(4):265-77.

23. Brasileiro SV, Orsini MR, Cavalcante JA, Bartholomeu D, Montiel JM, Costa PS, et al. Controversies Regarding the Psychometric Properties of the Brief COPE: The Case of the Brazilian-Portuguese Version “COPE Breve". PloS one. 2016;11(3):e0152233.

24. Perczek R, Carver CS, Price AA, Pozo-Kaderman C. Coping, mood, and aspects of personality in Spanish translation and evidence of convergence with English versions. J Pers Assess. 2000;74(1):63-87.

25. Kim Y, Seidlitz L. Spirituality moderates the effect of stress on emotional and physical adjustment. Pers Individ Dif. 2002;32(8):1377-90.

26. Muller L, Spitz E. Multidimensional assessment of coping: validation of the Brief COPE among French population. Encephale. 2003;29(6):507-18.

27. Ribeiro J, Rodrigues A. Questões acerca do coping: A propósito do estudo de adaptação do Brief Cope. Psicologia, Saúde \& Doenças. 2004;5(1):3-15.

28. Kapsou M, Panayiotou G, Kokkinos CM, Demetriou AG. Dimensionality of coping an empirical contribution to the construct validation of the brief-cope with a Greek-speaking sample. J Health Psychol. 2010;15(2):215-29.

29. Mohanraj R, Jeyaseelan V, Kumar S, Mani T, Rao D, Murray KR, et al. Cultural adaptation of the Brief COPE for persons living with HIV/AIDS in Southern India. AIDS Behav. 2015;19(2):341-51.

30. MacCallum RC, Widaman KF, Zhang S, Hong S. Sample size in factor analysis. Psychol Methods. 1999;4(1):84.

31. Afrasiabifar A, Karimi Z, Hassani P. Roy's Adaptation ModelBased patient education for promoting the adaptation of hemodialysis patients. Iran Red Crescent Med J. 2013;15(7):566.

32. Gurkan A, r Pakyuz S, Demir T. Stress coping strategies in 
hemodialysis and kidney transplant patients. Transplant Proc. 2015;47(5):1392-7.

33. Wild D, Grove A, Martin M, Eremenco S, McElroy S, VerjeeLorenz A, et al. Principles of good practice for the translation and cultural adaptation process for patient-reported outcomes (PRO) measures: report of the ISPOR Task Force for Translation and Cultural Adaptation. Value Health. 2005;8(2):94-104.

34. Yaghmale F. Content validity and its estimation. JME. 2009;3(1).

35. Zamanzadeh V, Ghahramanian A, Rassouli M, Abbaszadeh A, Alavi-Majd H, Nikanfar A-R. Design and implementation content validity study: development of an instrument for measuring patientcentered communication. J Caring Sci. 2015;4(2):165.

36. Kimberlin CL, Winterstein AG. Validity and reliability of measurement instruments used in research. Am J Health Syst Pharm. 2008;65(23):2276-84.

37. Gregory RJ. Psychological testing: History, principles, and applications: Allyn \& Bacon; 2004.

38. Mohammadbeigi A, Mohammadsalehi N, Aligol M. Validity and Reliability of the Instruments and Types of MeasurmentS in Health Applied Researches. JRUMS. 2015;13(12):1153-70.

39. Farmanbar R, Niknami S, Hidarnia A, Lubans DR. Psychometric properties of the Iranian version of the Behavioral Regulation in Exercise Questionnaire-2 (BREQ-2). Health Promot Perspect. 2011;1(2):95.

40. Lomax RG, Schumacker RE. A beginner's guide to structural equation modeling: Routledge Academic New York, NY; 2012.

41. Tabachnick BG, Fidell LS, Osterlind SJ. Using multivariate statistics. Boston: Allyin \& Bacon; 2001.

42. Bentler P, Stein J. Structural equation models in medical research. Stat Methods Med Res. 1992;1(2):159-81.

43. Byrne BM. Structural equation modeling with LISREL, PRELIS, and SIMPLIS: Basic concepts, applications, and programming: Psychology Press; 2013.

44. Nunnally JC, Bernstein IH, Berge JMt. Psychometric theory: JSTOR; 1994.

45. Streiner DL. Starting at the beginning: an introduction to coefficient alpha and internal consistency. J Pers Assess. 2003;80(1):99-103.

46. Streiner DL. A checklist for evaluating the usefulness of rating scales. Can J Psychiatry. 1993;38(2):140-8.

47. Polit DF, Beck CT. Essentials of nursing research: Appraising evidence for nursing practice: Lippincott Williams \& Wilkins; 2013.

48. Folkman S, Moskowitz JT. Coping: Pitfalls and promise. Annu Rev Psychol. 2004;55:745-74.

49. Yusoff MSB. The validity of the Malay Brief COPE in identifying coping strategies among adolescents in secondary school. Int Med J. 2011;18(1):29-33.

50. Yusoff MSB. A Multicenter Study on Validity of the ISO-Items Brief COPE in Identifying Coping Strategies among Medical Students. IMJ. 2010;177(4).

51. Monzani D, Steca P, Greco A, D’Addario M, Cappelletti E, Pancani L. The situational version of the Brief COPE: Dimensionality and relationships with goal-related variables. Eur J Psychol. 2015;11(2):295.

52. Dias C, Cruz JF, Fonseca AM. Anxiety and coping strategies in sport contexts: A look at the psychometric properties of Portuguese instruments for their assessment. Span J Psychol. 2009;12(01):33848.

53. Hagan TL, Fishbein JN, Nipp RD, Jacobs JM, Traeger L, Irwin KE, et al. Coping in Patients with Incurable Lung and Gastrointestinal Cancers: A Validation Study of the Brief COPE. J Pain Symptom Manage. 2016;53(1):131-8.

54. Egisdottir S, Gerstein LH, Cinarbas DC. Methodological issues in cross-cultural counseling research equivalence, bias, and translations. TCP. 2008;36(2):188-219. 